\title{
Integrating Depth of Invasion in T Classification Improves the Prognostic Performance of the American Joint Committee on Cancer Staging System for Laryngeal Squamous Cell Carcinoma: a Retrospective Multicenter Study
}

\author{
xueying wang \\ Harbin Medical University \\ kui cao \\ Harbin Medical University \\ erliang guo \\ Harbin Medical University \\ xionghui mao \\ Harbin Medical University \\ changming an \\ Chinese Academy of Sciences \\ lunhua guo \\ harbin medical university \\ cong zhang \\ harbin medical university \\ xiangguang yang \\ harbin medical university \\ ji sun
}

harbin medical university

weiwei yang

harbin medical university

xiaomei li

harbin medical university

Susheng Miao ( $\nabla$ drmiaosusheng@126.com )

Harbin Medical University Third Hospital: Tumor Hospital of Harbin Medical University

Research 
Keywords: Laryngeal squamous cell carcinoma (LSCC), American Joint Committee on Cancer (AJCC), Depth of invasion (DOI), Overall survival (OS), Relapse-free survival (RFS)

Posted Date: October 11th, 2021

DOl: https://doi.org/10.21203/rs.3.rs-957514/v1

License: (c) (1) This work is licensed under a Creative Commons Attribution 4.0 International License. Read Full License 


\section{Abstract}

Background: It has been recognized that depth of invasion (DOI) is closely associated with patient survival for all types of cancer. The purpose of this study was to determine the optimal threshold and prognostic value in laryngeal squamous carcinoma (LSCC). Most importantly, we evaluated the prognostic performance of five candidate modified T-classification models in patients with LSCC.

Methods: LSCC patients from Harbin Medical University Cancer Hospital and Chinese Academy of Medical Sciences Cancer Hospital were divided into training group $(n=412)$ and validation group $(n=147)$. The primary outcomes were overall survival (OS) and relapse-free survival (RFS), and the effect of DOI on prognosis was analyzed using a multivariable regression model. We identified the optimal model based on its simplicity, goodness of fit and Harrell's consistency index. Further independent testing was performed on the external validation queue. The nomograms was constructed to predict an individual's OS rate at one, three, and five years.

Results: In multivariate analysis, we found significant associations between DOI and OS (Depth of Medium-risk invasion HR, 2.631; $P<0.001$. Depth of high-risk invasion: HR, 5.287; $P<0.001$ ) and RFS(Depth of high-risk invasion: HR, 1.937; $P=0.016)$. Model 5 outperformed the American Joint Committee on Cancer (AJCC) staging system based on a low Akaike information criterion score, improvement in the concordance index, and Kaplan-Meier curves.

Conclusions: Inclusion of DOI in the current AJCC staging system can improve the differentiation of T classification in LSCC patients.

\section{Background}

Pathological features were included in the main T classification of head and neck squamous cell carcinoma (HNSCC) in the eighth edition of the Cancer Staging Manual of the Joint Council on Cancer (AJCC) [1]. The creation of a new staging system for high-risk human papillomavirus-associated oropharyngeal cancer (OPC) and inclusion of depth of invasion into oral cancer T staging are two of the most important aspects of this change. Incorporating these factors into clinical practice can help doctors, patients, and researchers clearly communicate disease status and improve prognosis and treatment options for HNSCC patients. Given the importance of pathological features, the College of American Pathologists recognized the need for standardization of specialized head and neck pathology synoptic reports $[2,3]$. This led to routine reporting of a defined set of pathological features, including Depth of invasion (DOI)[4-6].

LSCC is one of the most aggressive head and neck malignancies, and surgical removal of the primary tumor at the initial stage of diagnosis is the standard treatment at all stages of the disease. It should be noted that the need for postoperative adjuvant therapy depends on several recognized high risk factors, including: T3-4, N2-3, vascular invasion, peripheral nerve invasion, positive/insufficient incision margin, or lymphatic extravasation and other pathological features[7-9]. DOI has been widely studied as an 
indicator of poor prognosis of oral squamous cell carcinoma(OSCC), cholangiocarcinoma, colorectal cancer, melanoma and other malignant tumors[10]. In addition to OSCC, DOI is also included in AJCC cancer classifications such as cutaneous melanoma, cholangiocarcinoma, and cervical cancer[11-13]. The existing AJCC Staging Manual 8 (AJCC 8) TNM staging system considers the positive effects of primary lesion anatomical zoning and primary lesion size on LSCC. However, the current T staging may underestimate the influence of the depth of primary tumor invasion on the prognosis and treatment selection of LSCC. For example, patients with laryngeal cancer with an invasion depth of $2 \mathrm{~mm}$ had the same T-stage as patients with laryngeal cancer with an invasion depth of $10 \mathrm{~mm}$, although the latter had a worse clinical prognosis. Previously, Masayuki et al and Ye et al demonstrated DOI as a strong predictor of cervical lymph node metastasis in LSCC $[14,15]$. However, it is not clear whether DOI is an independent predictor of recurrent and survival of LSCC. In view of the above analysis, a more detailed description and inclusion of DOI may be beneficial for risk stratification of LSCC patients. This study aims to demonstrate the prognostic value of DOI for LSCC patients and, more importantly, to conceive a new staging system that can use DOI to improve the prognostic performance of LSCC. Here we compare the prognostic performance of multiple staging models, including the current AJCC and our newly constructed fivestaging system.

\section{Materials And Methods 2.1 Patients}

This is a multi-center retrospective study. We first collected 759 patients with LSCC diagnosed between January 2013 and December 2015 from the Department of Head, Neck, Nose and Throat Surgery, Harbin Medical University Tumor Hospital. Inclusion criteria: (1) pathologically proven LSCC; (2) resection of laryngeal cancer; (3) Complete clinical data such as laboratory examination and imaging examination. Exclusion criteria: (1) patients with recurrent primary tumor; (2) a history of preoperative chemotherapy and/or radiotherapy; (3) Patients with positive surgical margins. (4) patients with severe liver and kidney dysfunction; (5) Patients with severe heart disease. Clinical data (sex, age, BMI, alcohol and smoking history, tumor location, differentiation, T-stage and N-stage,TNM) and follow-up information (clinical outcome and survival) were collected through electronic medical record review. Patients were staged according to the AJCC8 staging system. Inclusion criteria require 5 or more years of postoperative followup and regular review. A total of 412 patients were enrolled after applying the criteria. Patients with LSCC who met the above inclusion and exclusion criteria were also admitted from Cancer Hospital of the Chinese Academy of Medical Sciences (Beijing, China) between December 2014 and December 2015. A total of 147 patients were assigned to the external validation cohort. Overall survival (OS) was defined as the period from pathological diagnosis to date of death, and relapse-free survival (RFS) was defined as the time from initial surgery to earliest evidence of recurrence. This study was reviewed and approved by the Cancer Hospital affiliated to Harbin Medical University and the Ethics Committee of the Cancer Hospital of Chinese Academy of Medical Sciences. The study was carried out in accordance with the 
principles of the Declaration of Helsinki and its amendments. All participants agreed to participate in the study.

\subsection{Histopathological analysis}

The specimens of the primary lesion were fixed with formalin and embedded in paraffin. The specimens were made into 3-4 micron serial sections and stained with hematoxylin and eosin (H\&E). The specimens were histopathologically diagnosed by two experienced pathologists, and they were not informed of the patient's clinical data. When there are major differences in the examination results, a third pathologist is required to participate. Measure the vertical distance from the deepest surface of the basement membrane (BM) to the deepest surface of the tumor using a microscope or a micrometer under an optical microscope magnification of 40 times. After multiple measurements, the average value is used as the DOI. The measured value is measured in millimeters.

\subsection{Model evaluation}

The proposed five models are compared with the current AJCC staging system. To develop a simple and accurate prognostic model. We use Akaike Information Criterion (AIC) to weigh the complexity of the model and the excellence of the fitting data. Next, Harrell's Concordance Index (C-index) was used to evaluate the predictive performance of the model[16]. Finally, Risk stratification was confirmed by visual inspection of Kaplane Meier curves. R software 4.0.3(Institute of Statistics and Mathematics, Vienna, Austria) was used to calculate the above evaluation index.

\subsection{Construction and evaluation of nomograms}

We combined the clinicopathological features with the DOI to construct a nomogram. We used the $\mathrm{C}$ index to evaluate the discriminative power of the nomogram and drew a calibration chart to evaluate the accuracy of the nomogram. We then compared the decision curve analysis between the AJCC eighth edition T staging and five newly constructed models.

\subsection{Statistical Analysis}

The primary outcome was OS from cancer diagnosis to death and the second outcome was RFS from LSCC diagnosis to disease recurrence or metastasis or cancer specific death, whichever came first. Outcomes were obtained during the follow-up period through January, 2020 via an established protocol. Postoperative patients were followed up at 3-6 months intervals for the first year and then annually.

We used the X-tile method to calculate the cut-off values of DOI before survival analyses. Using these cutoff values, we categorized patients in to three groups: low-, medium-, and high-risk groups. Traditional statistical method like the ROC and youden index method can calculate only one cutoff and therefore categorize participants into two subsets (low- or high-risk groups). Statisticians have noted that the significance of many prognostic analyses using these discrepancies by only one cut-off point cannot be confirmed in subsequent clinical studies. The algorithms in the X-tile method correct for this discrepancy. 
In the X-tile method, two cut-off points in survival analyses can dramatically increase the likelihood of finding an aberrantly low $p$-value.

We used univariate and multivariate Cox proportional hazards regression models to compare survival rate differences. We reported the corresponding hazard ratios (HR) and 95\% confidence intervals (Cls) for associations of variables with OS or RFS of the disease. The variables with statistical significance in the univariate analysis were included in the multivariate Cox regression model. The likelihood ratios backward stepwise method was used for the multivariate Cox regression analysis. All analyses were performed using R software 4.0.3(Institute of Statistics and Mathematics, Vienna, Austria). P $<0.05$ was considered statistically significant.

\section{Results}

\subsection{Baseline patient characteristics}

Table 1 summarizes the demographic and clinicopathological characteristics of the training and validation cohort. A total of 412 patients were included in the training set, including 336 men (81.6\%) for a total of 147 patients in the validation cohort. Two optimal truncation values were determined by $x$-tile method, which were 10.5 and 19.9 respectively. In the training set, gender $(P=0.006)$, age $(P=0.002)$, $B M I$ $(P=0.003)$, smoking $(P<0.001)$, alcohol $(P=0.002)$, and tumor location $(P<0.001)$ differences in $D O I$ (Table 1). 
Table 1

Summary data for LSCC DOI in the study patients (N $=412$ ).

\begin{tabular}{|llll|}
\hline Characteristic & No. (\%) & DOI (mm) & P \\
& & Mean & \\
\hline Gender & & & \\
\hline Male & $336(81.6)$ & 9.1 & 0.006 \\
\hline Female & $76(18.4)$ & 7.9 & \\
\hline Age (yr) & & & \\
\hline$<60$ & $223(54.1)$ & 9.1 & 0.002 \\
\hline$\geq 60$ & $189(45.9)$ & 8.6 & \\
\hline BMI (kg/m2) & & & \\
\hline$<24$ & $273(66.3)$ & 8.4 & 0.003 \\
\hline$\geq 24$ & $139(33.7)$ & 9.1 & \\
\hline Smoking & & & \\
\hline No & $114(27.7)$ & 8.8 & $<0.001$ \\
\hline Yes & $298(72.3)$ & 8.9 & \\
\hline Alcohol & & & \\
\hline No & $226(54.9)$ & 8.5 & 0.002 \\
\hline Yes & $186(45.1)$ & 9.0 & \\
\hline Initial Site & & & \\
\hline supraglottic & $215(52.2)$ & 8.7 & \\
\hline glottic larynx & $197(47.8)$ & 8.6 & \\
\hline Differentiation & & & \\
\hline Low-moderate & $268(65.0)$ & 8.3 & \\
\hline high & $114(41.0)$ & 8.7 & \\
\hline T-Stage & & & \\
\hline T1 & & & \\
\hline T2 & & & \\
\hline T3 & & & \\
\hline
\end{tabular}




\begin{tabular}{|llll|}
\hline Characteristic & No. (\%) & $\begin{array}{l}\text { DOI (mm) } \\
\text { Mean }\end{array}$ & P \\
\hline T4 & $16(3.9)$ & 7.6 & \\
\hline N-Stage & & & 0.718 \\
\hline N0 & $210(51.1)$ & 8.4 & \\
\hline N1 & $35(8.4)$ & 9.1 & \\
\hline N2 & $61(14.8)$ & 8.8 & \\
\hline N3 & $106(25.7)$ & 9.1 & \\
\hline TNM Stage & & & \\
\hline 1 & $120(29.1)$ & 7.7 \\
\hline 2 & $150(36.4)$ & 7.0 \\
\hline 3 & $71(17.2)$ & 7.4 \\
\hline 4 & $72(17.5)$ & 7.2 \\
\hline Depth(mm) & & \\
\hline $0.2-3.4$ & $86(20.9)$ & - \\
\hline $3.5-10.5$ & $250(60.7)$ & - \\
\hline $10.6-25.0$ & $76(18.4)$ & - \\
\hline
\end{tabular}

\subsection{Screening for independent prognostic factors}

Univariate and multivariate analyses were performed to consider the impact of clinicopathological features on overall survival and recurrence of LSCC. In univariate analysis, higher DOI predicted worse OS (Depth of Medium-risk invasion HR, 2.597; $\mathrm{P}<0.001$. Depth of high-risk invasion: HR, 4.478; $\mathrm{P}<0.001$ ) and RFS (Depth of high-risk invasion: $H R, 1.938 ; P=0.016)$. In addition, significant survival differences were found in BMI, degree of differentiation, T stage, $\mathrm{N}$ stage and TNM stage (Table 2). These important variables were included in the multivariate analysis to further screen out the important factors. In multivariate analysis, BMI(HR, 0.926; P = 0.005), and DOI (Depth of Medium-risk invasion HR, 2.631; P $<0.001$. Depth of high-risk invasion: HR, 5.287; $\mathrm{P}<0.001$ ) were significantly associated with OS (Table 2 ). We did not find clinically significant indicators in patients' RFS analysis (Table S1). 
Table 2

Overall survival analyses of 412 patients of LSCC (AJCC 8).

\begin{tabular}{|c|c|c|c|c|c|c|}
\hline \multirow[t]{2}{*}{ Items } & \multicolumn{3}{|c|}{ Univariable Analysis } & \multicolumn{3}{|c|}{ Multivariable Analysis } \\
\hline & HR & $95 \% \mathrm{Cl}$ & P-value & HR & $95 \% \mathrm{Cl}$ & P-value \\
\hline \multicolumn{7}{|l|}{ Gender } \\
\hline Male & ref & ref & ref & & & \\
\hline Female & 0.942 & $0.597-1.489$ & 0.799 & & & \\
\hline \multicolumn{7}{|l|}{ Age (yr) } \\
\hline$<60$ & ref & ref & ref & & & \\
\hline$\geq 60$ & 0.761 & $0.530-1.092$ & 0.138 & & & \\
\hline \multicolumn{7}{|l|}{ BMI (kg/m2) } \\
\hline$<24$ & ref & ref & ref & ref & ref & ref \\
\hline$\geq 24$ & 0.935 & $0.897-0.974$ & 0.001 & 0.926 & $0.878-0.977$ & 0.005 \\
\hline \multicolumn{7}{|l|}{ Smoking } \\
\hline No & ref & ref & ref & & & \\
\hline Yes & 1.589 & $0.999-2.523$ & 0.051 & & & \\
\hline \multicolumn{7}{|l|}{ Alcohol } \\
\hline No & ref & ref & ref & & & \\
\hline Yes & 1.243 & $0.862-1.792$ & 0.244 & & & \\
\hline \multicolumn{7}{|l|}{ Initial Site } \\
\hline supraglottic & ref & ref & ref & & & \\
\hline glottic larynx & 0.858 & $0.545-1.349$ & 0.506 & & & \\
\hline \multicolumn{7}{|l|}{ Differentiation } \\
\hline Low-moderate & ref & ref & ref & ref & ref & ref \\
\hline high & 0.521 & $0.326-0.834$ & 0.006 & 0.697 & $0.423-1.148$ & 0.156 \\
\hline \multicolumn{7}{|l|}{ T-Stage } \\
\hline T1 & ref & ref & ref & ref & ref & ref \\
\hline $\mathrm{T} 2$ & 1.507 & $0.957-2.372$ & 0.076 & 1.243 & $0.781-1.980$ & 0.359 \\
\hline T3 & 2.482 & $1.421-4.338$ & 0.001 & 1.812 & $1.006-3.261$ & 0.048 \\
\hline
\end{tabular}




\begin{tabular}{|c|c|c|c|c|c|c|}
\hline \multirow[t]{2}{*}{ Items } & \multicolumn{3}{|c|}{ Univariable Analysis } & \multicolumn{3}{|c|}{ Multivariable Analysis } \\
\hline & HR & $95 \% \mathrm{Cl}$ & P-value & HR & $95 \% \mathrm{Cl}$ & P-value \\
\hline T4 & 1.658 & 0.705-3.896 & 0.246 & 1.189 & $0.502-2.814$ & 0.694 \\
\hline \multicolumn{7}{|l|}{ N-Stage } \\
\hline NO & ref & ref & ref & ref & ref & ref \\
\hline N1 & 1.464 & $0.741-2.895$ & 0.272 & 1.087 & $0.532-2.223$ & 0.817 \\
\hline N2 & 2.859 & $1.854-4.410$ & $<0.001$ & 2.357 & $1.465-3.791$ & $<0.001$ \\
\hline N3 & 1.125 & $0.682-1.858$ & 0.645 & 1.111 & $0.668-1.849$ & 0.683 \\
\hline \multicolumn{7}{|c|}{ TNM Stage } \\
\hline 1 & ref & ref & ref & ref & ref & ref \\
\hline 2 & 1.144 & $0.715-1.832$ & 0.057 & 2.373 & $1.455-3.871$ & 0.372 \\
\hline 3 & 1.118 & $0.641-1.949$ & 0.031 & 2.583 & $1.405-4.748$ & 0.002 \\
\hline 4 & 1.646 & $0.950-2.849$ & $<0.001$ & 7.536 & $3.908-14.532$ & $<0.001$ \\
\hline \multicolumn{7}{|c|}{ Depth (mm) } \\
\hline $0.2-10.5$ & ref & ref & ref & & & \\
\hline 10.5-19.9 & 2.597 & $1.696-3.978$ & $<0.001$ & 2.631 & $1.711-4.046$ & $<0.001$ \\
\hline $19.9-25.0$ & 4.478 & 2.709-7.401 & $<0.001$ & 5.287 & $3.131-8.927$ & $<0.001$ \\
\hline
\end{tabular}

\subsection{Construction and evaluation of prediction models}

We constructed five candidate T classification models for evaluation based on AJCC 8 (Table 3). First, survival curves of LSCC patients were drawn based entirely on AJCC 8 T staging (Figure 1A). The distinction between stage T2 and T3 patients is its greatest weakness. Further, we combined AJCC and DOI to construct model 1 (Figure 1B). Although AIC and C-index were superior to AJCC 8, it still did not differentiate between stage T2 and T3 patients. Models 2 and 3 showed superior performance in predicting the prognosis of patients at stage $\mathrm{T} 1$ and $\mathrm{T} 4$, but unfortunately did not address the existing problems (Figure 1A and 1D). To solve this problem, we continue to build model 4 (Figure 1E). Model 4 demonstrated a good ability to stratify risk in patients of different age groups. We tried to find a new staging system that could go beyond model 4 , but model 5 did not perform very well (Figure 1F), either in terms of risk stratification for early disease or in terms of both measures. After comprehensively considering the identification ability of patients with different stratification and referring to the two evaluation indexes AIC and C index, we believe that Model 4 is the best compared with the other four 
models. We continued to evaluate the layering capabilities of AJCC and model 4 on RFS. Compared with AJCC, model 4 showed better predictive ability in both AIC, C-index and Kaplan- Meier stratification (Figure 2A and 2B). 
Table 3

T classifications for primary LSCC in the eighth edition of the AJCC cancer staging manual and our five candidate models.

\section{T Supraglottic \\ classifification \\ Glottic larynx}

AJCC T1

The tumor was confined to a subregion of the glottis and the vocal cords were normally active

$\begin{array}{ll} & \begin{array}{l}\text { glottis and the vocal cords were normally } \\ \text { active }\end{array} \\ \text { T2 } & \begin{array}{l}\text { The tumor invades more than one adjacent } \\ \text { subregion of the glottic region or beyond the } \\ \text { glottic region, without laryngeal fixation }\end{array}\end{array}$

T3 The tumor is localized in the larynx, with vocal cord fixation and/or invasion of any of the following sites: posterior annulus, anterior epiglottis space, paraportal space, and/or thyroid cartilage plate

The tumor is confined to the vocal cord and the vocal cord is normally active

Invasion of supratiglottic and/or subglottic areas, and/or limitation of vocal cord movement

T3

Moderately advanced or very advanced local disease

The tumor is localized in the larynx, accompanied by vocal cord fixation and/or invasion of the paraventricular space, and/or thyroid cartilage

$\mathrm{T} 4$

Moderately advanced local disease neoplasms

$\mathrm{T} 4 \mathrm{a}$ invade through the thyroid cartilage and/or invade the extralyngeal tissue

Moderately advanced or very advanced local disease

Moderately advanced local disease neoplasms invade through the thyroid cartilage and/or invade the extralyngeal tissue

T4b Very advanced local disease tumors invade the prevertebral fascia, surround the carotid artery or invade mediastinal structures

Very advanced local disease tumors invade the prevertebral fascia, surround the carotid artery or invade mediastinal structures

\begin{tabular}{|c|c|c|}
\hline Model 1 T1 & AJCC T1, DOI $\leq 10.5 \mathrm{~mm}$ & AJCC T1, DOI $\leq 10.5 \mathrm{~mm}$ \\
\hline T2 & $\begin{array}{l}\text { AJCC T1, DOI>10.5mm; or AJCC T2, DOI } \leq 19.9 \\
\mathrm{~mm}\end{array}$ & $\begin{array}{l}\text { AJCC T1, DOl> } 10.5 \mathrm{~mm} \text {; or AJCC } \\
\text { T2, DOI } \leq 19.9 \mathrm{~mm}\end{array}$ \\
\hline T3 & $\begin{array}{l}\text { AJCC T2, DOl>19.9 mm; or AJCC T3, } \\
\text { DOI } \leq 19.9 \mathrm{~mm}\end{array}$ & $\begin{array}{l}\text { AJCC T2, DOl>19.9 mm; or AJCC } \\
\text { T3, DOI } \leq 19.9 \mathrm{~mm}\end{array}$ \\
\hline $\mathrm{T} 4$ & AJCC T3, DOI> $19.9 \mathrm{~mm}$; or AJCC T4 & $\begin{array}{l}\text { AJCC T3, DOI>19.9mm; or AJCC } \\
\text { T4 }\end{array}$ \\
\hline Model $2 \mathrm{~T} 1 \mathrm{a}$ & AJCC T1, DOI $\leq 10.5 \mathrm{~mm}$ & AJCC T1, DOI $\leq 10.5 \mathrm{~mm}$ \\
\hline $\mathrm{T} 1 \mathrm{~b}$ & AJCC T1, DOI>10.5mm & AJCC T1, DOI>10.5mm \\
\hline T2 & AJCC T2, DOI $\leq 19.9 \mathrm{~mm}$ & AJCC T2, DOI $\leq 19.9 \mathrm{~mm}$ \\
\hline T3 & $\begin{array}{l}\text { AJCC T2, DOl>19.9 mm; or AJCC T3-4, } \\
\text { DOI } \leq 19.9 \mathrm{~mm}\end{array}$ & $\begin{array}{l}\text { AJCC T2, DOI>19.9 mm; or AJCC } \\
\text { T3-4, DOI } \leq 19.9 \mathrm{~mm}\end{array}$ \\
\hline T4 & AJCC T3-4, DOI>19.9mm & AJCC T3-4, DOI>19.9mm \\
\hline
\end{tabular}




\begin{tabular}{|c|c|c|}
\hline $\begin{array}{l}\text { T } \\
\text { classifification }\end{array}$ & Supraglottic & Glottic larynx \\
\hline Model 3 T1 & AJCC T1, DOI $\leq 10.5 \mathrm{~mm}$ & AJCC T1, DOI $\leq 10.5 \mathrm{~mm}$ \\
\hline $\mathrm{T} 2$ & $\begin{array}{l}\text { AJCC T1, DO>10.5mm; AJCC T2, DOI } \leq 19.9 \\
\mathrm{~mm}\end{array}$ & $\begin{array}{l}\text { AJCC T1, DO> } 10.5 \mathrm{~mm} ; \text { AJCC T2, } \\
\text { DOl } \leq 19.9 \mathrm{~mm}\end{array}$ \\
\hline T3 & $\begin{array}{l}\text { AJCC T2, DOl>19.9 mm; or AJCC T3-4, } \\
\text { DOl } \leq 19.9 \mathrm{~mm}\end{array}$ & $\begin{array}{l}\text { AJCC T2, DOI>19.9 mm; or AJCC } \\
\text { T3-4, DOI } \leq 19.9 \mathrm{~mm}\end{array}$ \\
\hline T4 & AJCC T3-4, DOI>19.9mm & AJCC T3-4, DOI>19.9mm \\
\hline Model 4 T1a & AJCC T1, DOI $\leq 10.5 \mathrm{~mm}$ & AJCC T1, DOI $\leq 10.5 \mathrm{~mm}$ \\
\hline T1b & AJCC T1, DOI>10.5mm & AJCC T1, DOI>10.5mm \\
\hline $\mathrm{T} 2$ & AJCC T2, DOI $\leq 19.9 \mathrm{~mm}$ & AJCC T2, DOI $\leq 19.9 \mathrm{~mm}$ \\
\hline T3 & $\begin{array}{l}\text { AJCC T2, DOl>19.9 mm; or AJCC T3, } \\
\text { DOI } \leq 19.9 \mathrm{~mm}\end{array}$ & $\begin{array}{l}\text { AJCC T2, DOl> } 19.9 \mathrm{~mm} \text {; or AJCC } \\
\text { T3, DOI } \leq 19.9 \mathrm{~mm}\end{array}$ \\
\hline T4 & AJCC T3, DOI> $19.9 \mathrm{~mm}$; or AJCC T4 & $\begin{array}{l}\text { AJCC T3, DOI>19.9mm; or AJCC } \\
\text { T4 }\end{array}$ \\
\hline Model 5 T1 & AJCC T1-2, DOI $\leq 10.5 \mathrm{~mm}$ & AJCC T1-2, DOI $\leq 10.5 \mathrm{~mm}$ \\
\hline T2 & $\begin{array}{l}\text { AJCC T1, DOl>10.5mm; or AJCC T2, } \\
10.5 \mathrm{~mm}<\mathrm{DOl} \leq 19.9 \mathrm{~mm}\end{array}$ & $\begin{array}{l}\text { AJCC T1, DOI> } 10.5 \mathrm{~mm} \text {; or AJCC } \\
\text { T2, } 10.5 \mathrm{~mm}<\text { DOl } \leq 19.9 \mathrm{~mm}\end{array}$ \\
\hline Т3 & $\begin{array}{l}\text { AJCC T2, DOl>19.9mm; or AJCC T3-4, } \\
\text { DOK<19.9mm }\end{array}$ & $\begin{array}{l}\text { AJCC T2, DOI> } 19.9 \mathrm{~mm} \text {; or AJCC } \\
\text { T3-4, DOl< } 19.9 \mathrm{~mm}\end{array}$ \\
\hline $\mathrm{T} 4$ & AJCC T3-4, DOI>19.9mm & AJCC T3-4, DOI>19.9mm \\
\hline
\end{tabular}

In the external validation cohort, model 4 is also a good description of the new T-classification and OS/RFS functionality. AIC and C-index even exceeded the training cohort (Figure 2C and 2D).

\subsection{Nomograms construction}

In order to further improve the accuracy of the prediction, we constructed a new nomogram based on the $\mathrm{DOI}$ and other clinical indicators (Figure $3 \mathrm{~A}$ ). The nomogram C-index is 0.722 By calculating the total score, oncologists can easily obtain the probability of OS predicted by the nomogram of a single patient. We also use the calibration curve to evaluate the model's prediction accuracy (Figure 3B). The results show that the prediction calibration curve of the three calibration points in 1,3 , and 5 years is close to the standard curve, which indicates that the model has good predictive performance. In addition, we also use the decision curve analysis (DCA) to evaluate the reliability of the model (Figure $3 \mathrm{C}$ ). It can be seen that model 4 is significantly higher than the limit curve and other models including AJCC 8 , so it has good reliability. 


\section{Discussion}

This study addresses the impact of DOI on laryngeal cancer prognosis, on the other hand, formulates two optimal DOI cut-off values for determining LSCC risk stratification. Most importantly, we construct a new staging system based on AJCC. We find that the inclusion of DOI in the current AJCC staging system provides better risk stratification in the T classification for OS.

LSCC staging with good predictive performance is the basis for personalized treatment of patients, communication with patients about prognosis and research on improving prognosis[17]. There are reasons to think that it is necessary to include DOI in the T class. A large number of data have shown that DOI can better reflect tumor invasion potential compared with tumor diameter[18]. Previously, the AJCC developed a new tumor-size staging system combined with DOI specifically for the poor prognostic performance of t-classification in many OSCC patients[19, 20]. We constructed five new LSCC models based on AJCC version 8, and evaluated the application value of the models from three aspects: the complexity of the models, the superiority of fitting data and the accuracy of prediction. Compared with existing AJCC stages, model 4 presented more comprehensive clinical implications and was relatively simple. In addition, we also show good predictive performance when applying the model to external validation queues.

Nomograms can predict tumor prognosis through simple visualization. Therefore, we developed a visual line-map based on the new model. The correlation between scores and clinical outcomes is demonstrated by an intuitive Nomu diagram, and the probability of outcome events can be easily calculated by clinicians. In our model, individual scores for each patient were calculated based on nomu's diagram and patients were successfully stratified at risk. For patients with high score (high-risk patients), traditional surgical methods may not achieve satisfactory results, so postoperative treatment can be considered as an alternative to adjuvant therapy.

Due to the obvious prognostic value of the depth of infiltration, DOI has been included in routine pathological reports after communication between the hospital and the pathology department. The possibility of evaluating DOI in HE staining sections, along with the high reproducibility, is simple and feasible for pathologists at no additional cost, making the newly constructed TNM staging system easy to apply for routine use.

Our study was the first to assess the prognosis of LSCC patients with DOI as the sole risk factor and creatively included DOI as a pathological feature in the AJCC staging system. In addition, the study included a large sample size and a multicenter design, which enhanced the reliability of our results. However, some limitations must be recognized. First, it was a retrospective study and may not be applicable to subglottic laryngeal cancer. Secondly, there may be differences between the two institutions in terms of differences between pathologists used to evaluate DOI. These limitations may need to be addressed in future studies. In conclusion, our results suggest that the prognostic information provided by DOI reinforces the clinical application of the current AJCC T classification for predicting the prognosis of patients with LSCC. 


\section{Conclusions}

In the AJCC staging system, LSCC lacks evidence for DOI classification. Our study is the first to include DOI in an LSCC staging system and assess its importance. DOI is a valuable indicator of clinicopathology that can be routinely incorporated into clinical practice and seems worthy of implementation in staging systems. It is necessary to initiate prospective studies, preferably multi-institutional studies, to further introduce TNM-DOI staging as part of routine prediction and treatment decisions for LSCC.

In LSCC, there is a lack of evidence that DOI influences patient outcomes. Our study is the first to report DOI as an independent factor influencing OS and RFS in LSCC patients. In addition, we emphasized the importance of DOI parameters as part of the new staging system for LSCC. As a valuable indicator of patient risk stratification, $\mathrm{DOI}$ can be routinely incorporated into clinical practice as part of routine prediction and treatment decisions for LSCC.

\section{Abbreviations}

HNSCC: head and neck squamous cell carcinoma ; LSCC: laryngeal squamous carcinoma; OS: overall survival; RFS: relapse-free survival; DOI: depth of invasion; AJCC: American Joint Committee on Cancer; OPC: oropharyngeal cancer; OSCC: oral squamous cell carcinoma; H\&E: hematoxylin and eosin; AIC: Akaike Information Criterion; C-index: Concordance Index; HR: hazard ratios; Cls: confidence intervals; DCA: decision curve analysis;

\section{Declarations}

\section{Ethics approval and consent to participate}

The study was approved by the Ethics Committee of Harbin Medical University and the Chinese Academy of Medical Sciences

\section{Consent for publication}

All authors consent for publication

\section{Availability of data and materials}

The data used to support the findings of this study are available from the corresponding author upon request

\section{Competing interests}


The authors declare that they have no competing interests.

\section{Funding}

This work was supported by Postdoctoral Scientific Research Developmental Fund of Heilongjiang Province (LBH-Q18088).

\section{Authors' contributions}

$\mathrm{XW}$ and $\mathrm{KC}$ conceptualized the project, all data analysis and wrote the first draft of the manuscript. EG, $X M, C A, L G, C Z, X Y, J S, W Y$ contributed to processing, analysis, and interpretation of the data. XL and SM contributed to guide the data analysis, and manuscript writing. All authors contributed to the article and approved the submitted version.

\section{Acknowledgments}

We would like show sincere appreciation to the editors and reviewers for critical comments on this article.

\section{References}

1. Lydiatt WM, Patel SG, O'Sullivan B, et al. Head and Neck cancers-major changes in the American Joint Committee on cancer eighth edition cancer staging manual. CA Cancer J Clin. 2017;67(2):12237.

2. Yunker WK, Matthews TW, Dort JC. Making the most of your pathology: standardized histopathology reporting in head and neck cancer. J Otolaryngol Head Neck Surg. 2008;37(1):48-55.

3. King B, Corry J. Pathology reporting in head and neck cancer-snapshot of current status. Head Neck. 2009;31(2):227-31. discussion 32-3.

4. International Consortium for Outcome Research in $\mathrm{H}$. Neck C, Ebrahimi A, et al. Primary tumor staging for oral cancer and a proposed modification incorporating depth of invasion: an international multicenter retrospective study. JAMA Otolaryngol Head Neck Surg. 2014;140(12):1138-48.

5. Liao CT, Lee LY, Hsueh C, et al. Tumor Depth of Invasion (Tumor $>4 \mathrm{~cm} /$ Depth $>10 \mathrm{~mm}$ and Depth $>$ $20 \mathrm{~mm}$ ) and Through Cortex/Skin Invasion are Both Valid Criteria for Classifying Tumors as pT4a in AJCC 2018 Oral Cavity Cancer Staging System. Ann Surg Oncol. 2019;26(11):3663-72.

6. Tam S, Amit M, Zafereo M, et al. Depth of invasion as a predictor of nodal disease and survival in patients with oral tongue squamous cell carcinoma. Head Neck. 2019;41(1):177-84.

7. Peters LJ, Goepfert H, Ang KK, et al. Evaluation of the dose for postoperative radiation therapy of head and neck cancer: first report of a prospective randomized trial. Int J Radiat Oncol Biol Phys. 1993;26(1):3-11. 
8. Bernier J, Domenge C, Ozsahin M, et al. Postoperative irradiation with or without concomitant chemotherapy for locally advanced head and neck cancer. N Engl J Med. 2004;350(19):1945-52.

9. Cooper JS, Pajak TF, Forastiere AA, et al. Postoperative concurrent radiotherapy and chemotherapy for high-risk squamous-cell carcinoma of the head and neck. N Engl J Med. 2004;350(19):1937-44.

10. Lau L, Eu D, Loh T, et al. Histopathologic prognostic indices in tongue squamous cell carcinoma. Eur Arch Otorhinolaryngol. 2020.

11. Park JY, Kim SY, Shin DH, et al. Validation of the T category for distal cholangiocarcinoma: Measuring the depth of invasion is complex but correlates with survival. Ann Diagn Pathol. 2020;46:151489.

12. Tomar AS, Finger PT, Gallie B, et al. A Multicenter, International Collaborative Study for American Joint Committee on Cancer Staging of Retinoblastoma: Part I: Metastasis-Associated Mortality. Ophthalmology. 2020.

13. Isom C, Wheless L, Hooks MA, et al. Early Melanoma Nodal Positivity and Biopsy Rates Before and After Implementation of the 7th Edition of the AJCC Cancer Staging Manual. JAMA Dermatol. 2019;155(5):572-7.

14. Ye LL, Rao J, Fan XW, et al. The prognostic value of tumor depth for cervical lymph node metastasis in hypopharyngeal and supraglottic carcinomas. Head Neck. 2019;41(7):2116-22.

15. Tomifuji M, Imanishi Y, Araki K, et al. Tumor depth as a predictor of lymph node metastasis of supraglottic and hypopharyngeal cancers. Ann Surg Oncol. 2011;18(2):490-6.

16. Harrell FE Jr, Califf RM, Pryor DB, et al. Evaluating the yield of medical tests. JAMA. 1982;247(18):2543-6.

17. Friedland $\mathrm{PL}$, Bozic $B$, Dewar J, et al. Impact of multidisciplinary team management in head and neck cancer patients. Br J Cancer. 2011;104(8):1246-8.

18. Dirven R, Ebrahimi A, Moeckelmann N, et al. Tumor thickness versus depth of invasion - Analysis of the 8th edition American Joint Committee on Cancer Staging for oral cancer. Oral Oncol. 2017;74:30-3.

19. Howaldt HP, Kainz M, Euler B, et al. Proposal for modification of the TNM staging classification for cancer of the oral cavity. DOSAK J Craniomaxillofac Surg. 1999;27(5):275-88.

20. Yuen AP, Lam KY, Wei WI, et al. A comparison of the prognostic significance of tumor diameter, length, width, thickness, area, volume, and clinicopathological features of oral tongue carcinoma. Am J Surg. 2000;180(2):139-43.

\section{Figures}


A
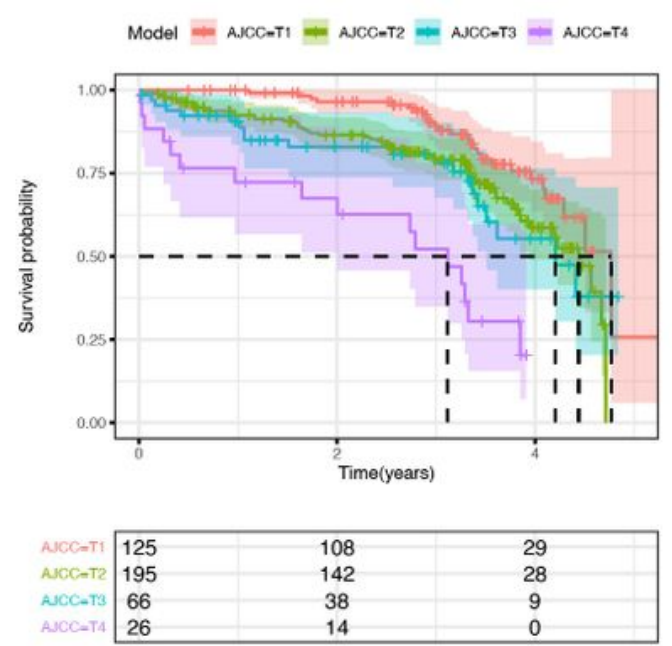

C
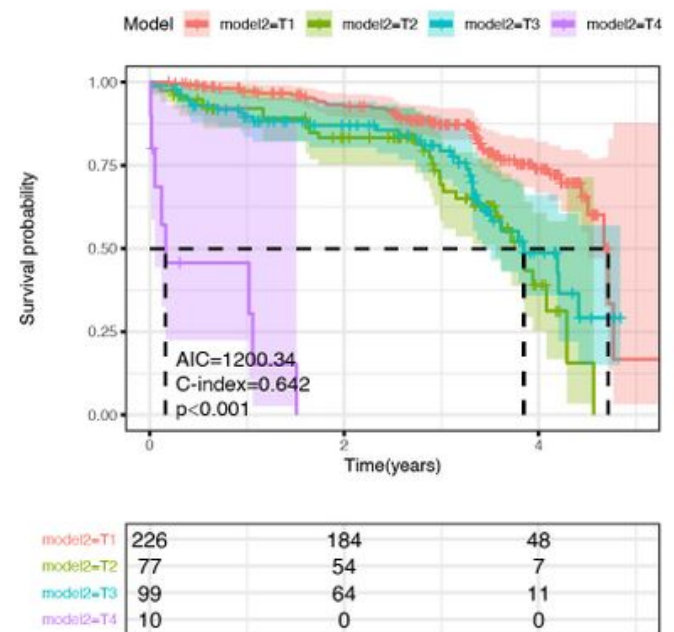

E

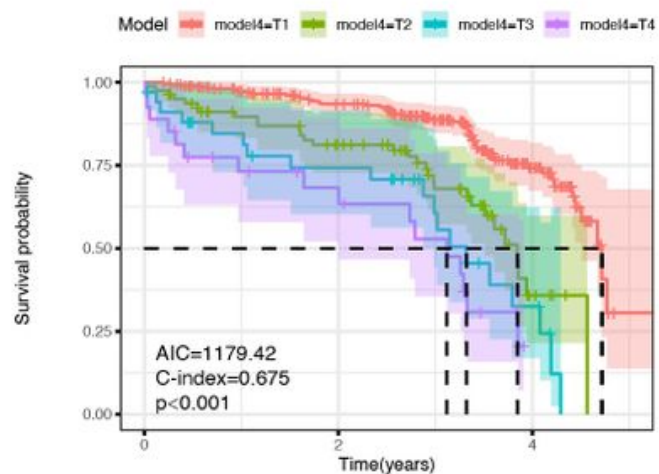

\begin{tabular}{l|c|c|c|}
\hline modelit-T1 & 271 & 213 & 56 \\
model4-T2 & 80 & 54 & 5 \\
model4-T3 & 34 & 21 & 5 \\
modelit-T4 & 27 & 14 & 0 \\
\hline
\end{tabular}

B

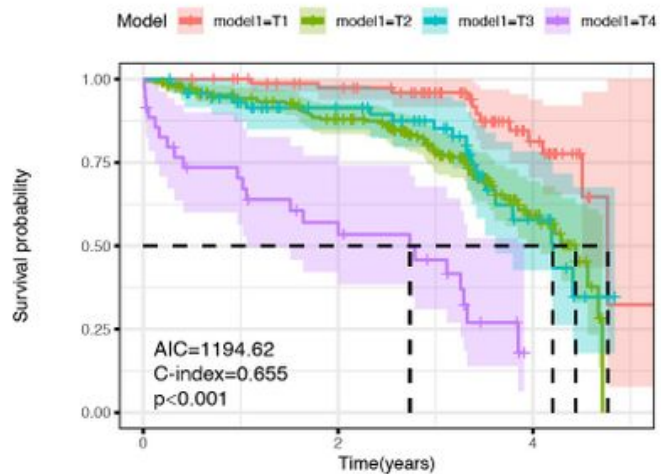

\begin{tabular}{|c|c|c|c|}
\hline modelite $\mathrm{T1}$ & 84 & 73 & 24 \\
\hline model1-T2 & 218 & 164 & 31 \\
\hline models= T3 & 75 & 49 & 11 \\
\hline modely $=T 4$ & 35 & 16 & 0 \\
\hline
\end{tabular}

D

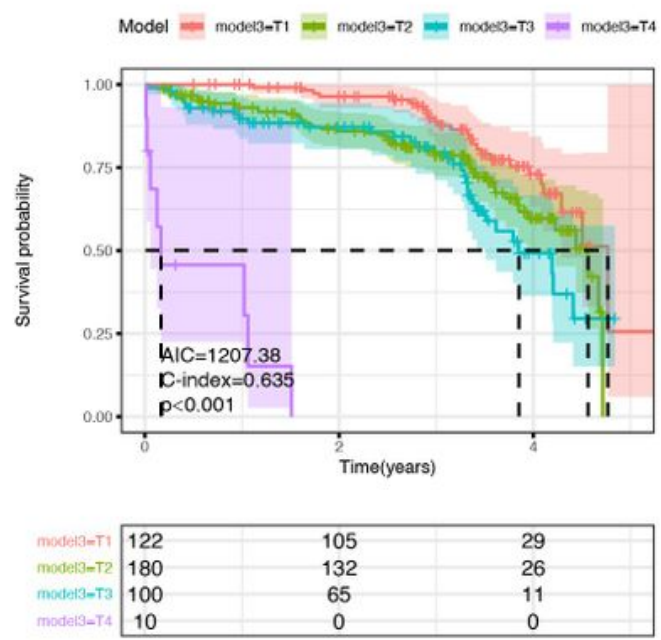

F

Model $=$ model5-T1 $=$ model5-T2 $=$ model5-T3 $=$ model5-T4

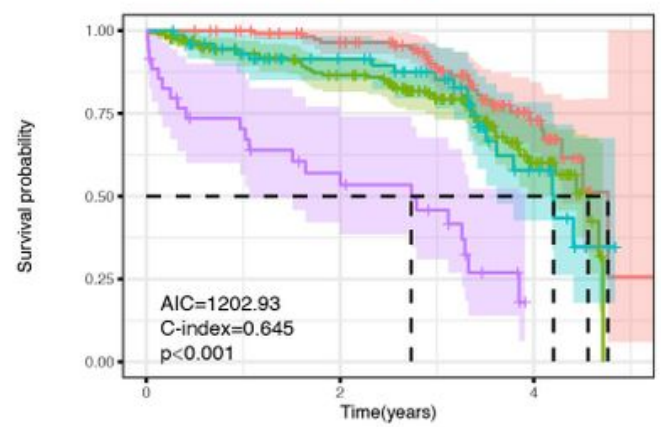

\begin{tabular}{l|ccc|}
\cline { 2 - 4 } model5-T1 & 122 & 105 & 29 \\
modal5-T2 & 180 & 132 & 26 \\
model5-T3 & 75 & 49 & 11 \\
models-T4 & 35 & 16 & 0 \\
\cline { 2 - 4 } & & &
\end{tabular}

\section{Figure 1}

KaplaneMeier plots demonstrating the ability of different staging systems to describe the primary tumor T classifications and OS in the study patients with LSCC. (A) Current AJCC T staging. (B-F) Models 1 (B), 2 (C), 3 (D), 4 (E) and 5 (F) in our proposed system. AJCC, American Joint Committee on Cancer; LSCC, laryngeal squamous carcinoma; AIC, Akaike information criterion; C-index: Concordance Index. 
A

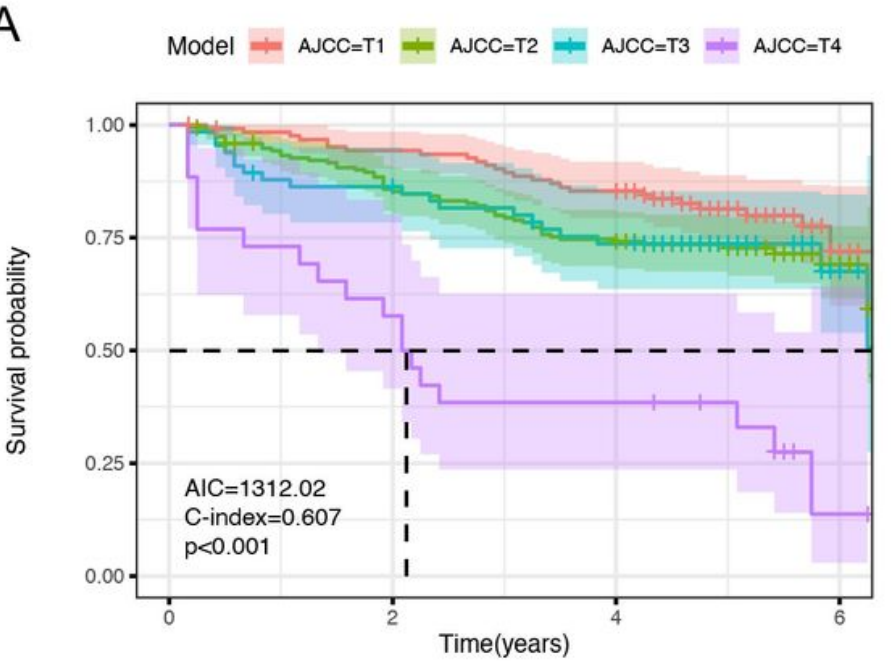

\begin{tabular}{l|c|c|c|c|}
\cline { 2 - 5 } A $N C C=T 1$ & 125 & 116 & 105 & 10 \\
A.JCC $=$ T2 & 195 & 162 & 141 & 20 \\
A.JCC=T3 & 66 & 56 & 46 & 7 \\
A.JCC=T4 & 26 & 15 & 10 & 1 \\
\cline { 2 - 5 } & & & &
\end{tabular}

C

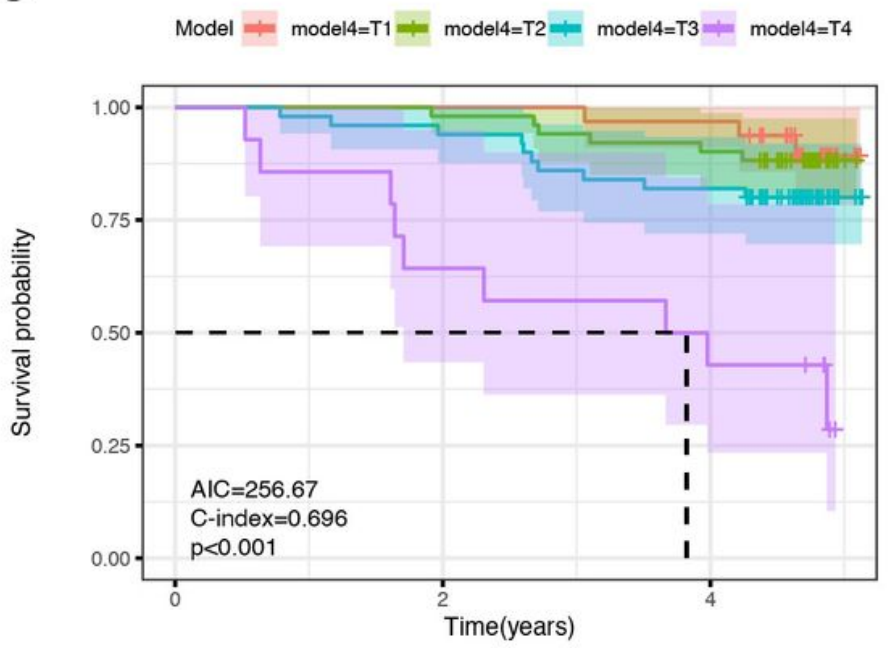

\begin{tabular}{l|l|c|c|}
\cline { 2 - 4 } model $4=\mathrm{T} 1$ & 32 & 32 & 31 \\
model $4=\mathrm{T} 2$ & 51 & 50 & 46 \\
model $4=\mathrm{T} 3$ & 50 & 47 & 41 \\
model $4=\mathrm{T} 4$ & 14 & 9 & 6 \\
\cline { 2 - 4 } & & &
\end{tabular}

B

Model $=$ model $4=\mathrm{T} 1=$ model $4=\mathrm{T} 2=\operatorname{model} 4=\mathrm{T} 3=$ model $4=\mathrm{T} 4$

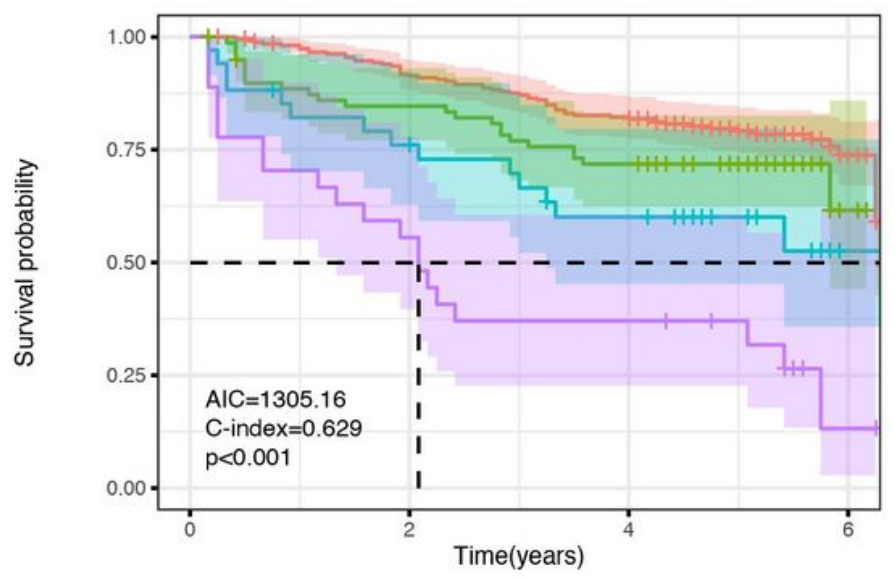

\begin{tabular}{l|c|c|c|c|}
\cline { 2 - 5 } model $4=\mathrm{T} 1$ & 271 & 243 & 218 & 33 \\
model $4=\mathrm{T} 2$ & 80 & 66 & 56 & 2 \\
model $4=\mathrm{T} 3$ & 34 & 25 & 18 & 2 \\
model $4=\mathrm{T} 4$ & 27 & 15 & 10 & 1 \\
\cline { 2 - 5 } & & & &
\end{tabular}

D

Model $\doteqdot$ model $4=1=$ model $4=2=$ model $4=3=$ model $4=4$

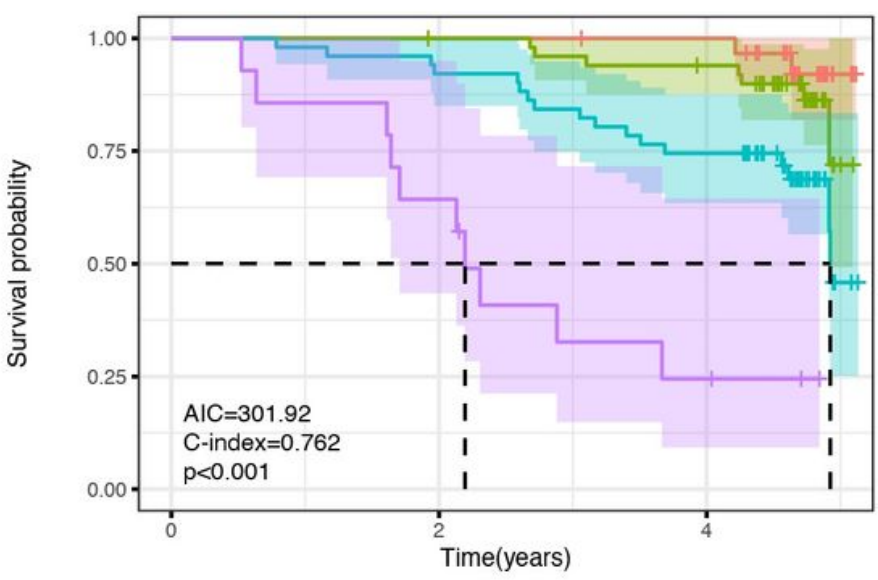

\begin{tabular}{l|l|c|c|}
\cline { 2 - 4 } model $4=1$ & 31 & 31 & 30 \\
mode $4=2$ & 51 & 50 & 46 \\
mode $4=3$ & 51 & 47 & 38 \\
model $4=4$ & 14 & 9 & 3 \\
\cline { 2 - 5 } & & &
\end{tabular}

\section{Figure 2}

Kaplane-Meier plots demonstrating the ability of different staging systems to describe the primary tumor T classifications and OS/RFS in training and validation cohorts. Kaplan-Meier survival plots for RFS by AJCC T staging (A) and Models 4 (B), respectively. In the validation queue, Kaplan-Meier survival plots for OS (C) and RFS (D) by models 4, respectively. AJCC, American Joint Committee on Cancer; LSCC, laryngeal squamous carcinoma; AIC, Akaike information criterion; C-index: Concordance Index. 

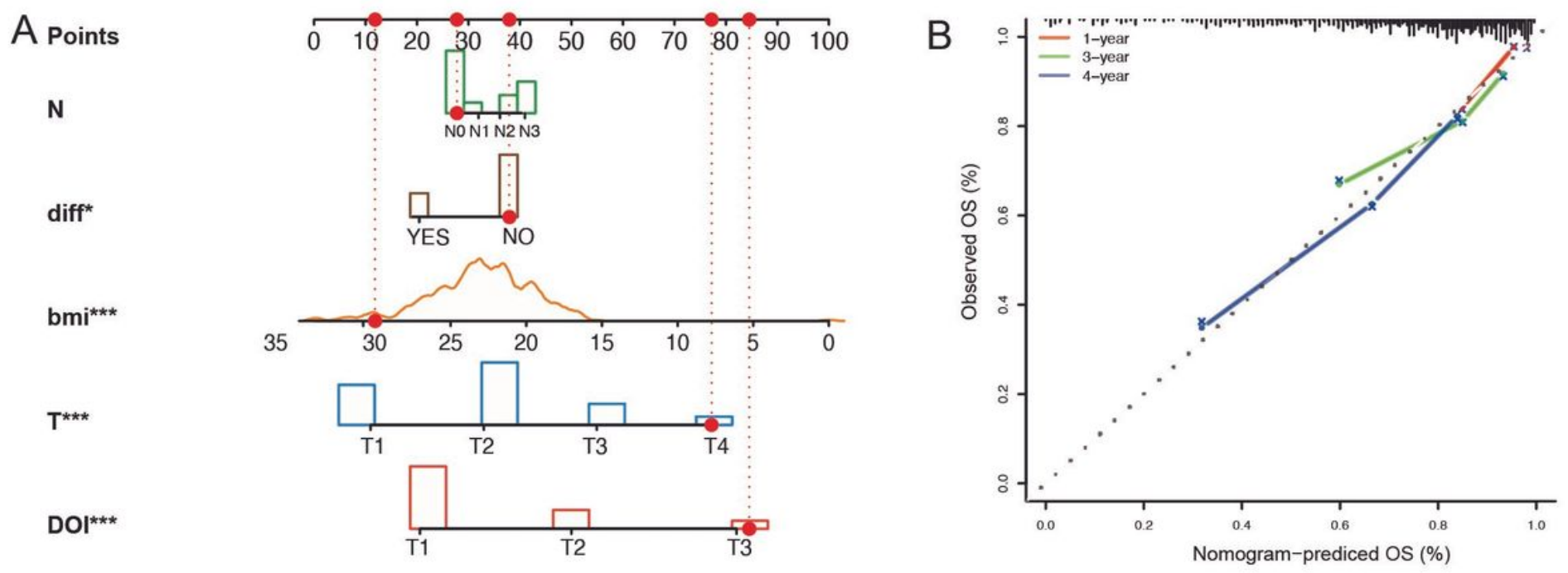

Total points
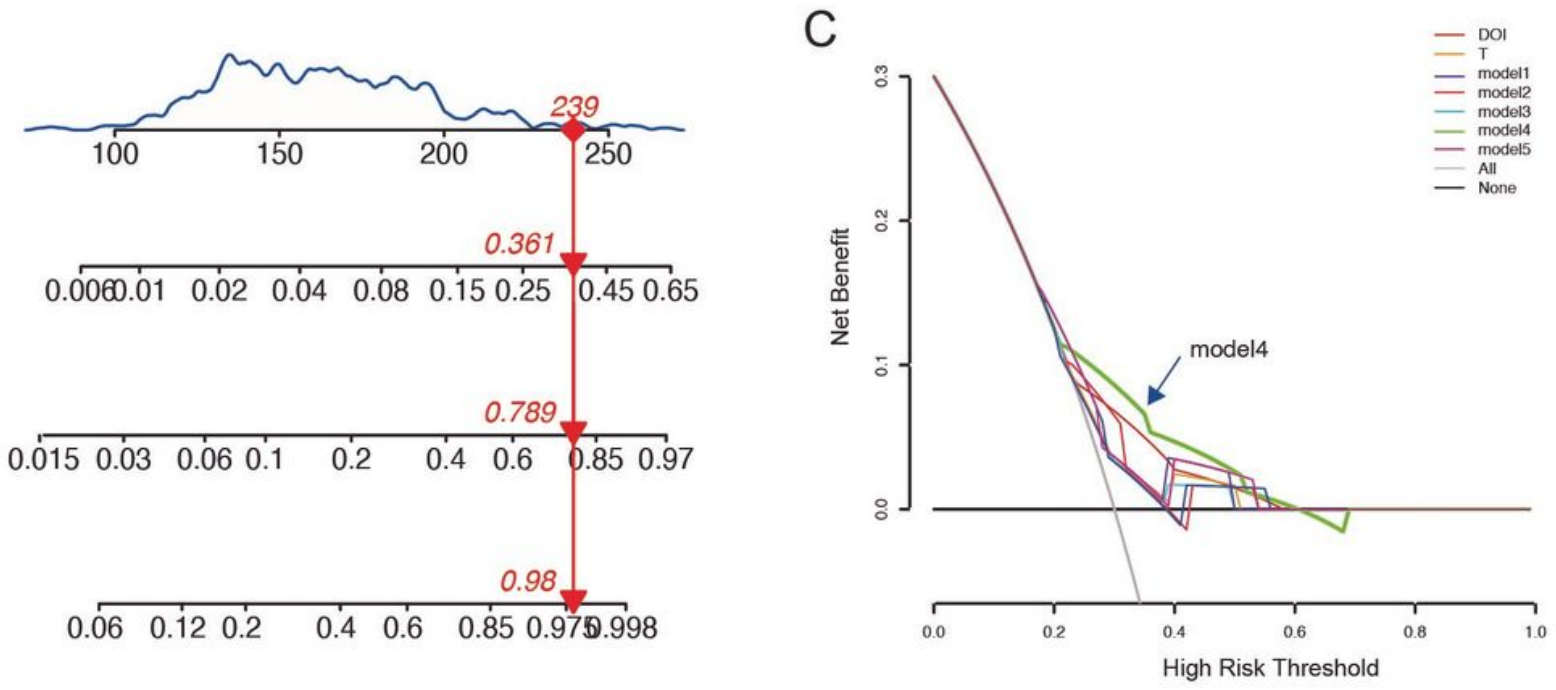

Figure 3

Establishment and evaluation of nomogram model. (A) Nomogram based on clinicopathological features for the prediction of 1-, 3- and 5-year OS. (B) Calibration curves of the nomogram for 1, 3 and 5 years. (C) Decision curve analysis of the nomograms based on clinicopathological features. ${ }^{*} P<0.05,{ }^{*} P<0.01$, $\star \star * P<0.001$.

\section{Supplementary Files}

This is a list of supplementary files associated with this preprint. Click to download.

- TableS1.docx 